\title{
A Consolidated and Comparative Analysis of Bellman Ford, DSR, OLSR and DREAM Routing Protocols for MANET: A Survey
}

\author{
Ashish Gupta \\ Department of Computer Science and Engineering, Mahamaya College of Agricultural Engineering and Technology, \\ Ambedkar Nagar (Uttar Pradesh)-224122, India
}

\begin{abstract}
MANET describes distributed, wireless, mobile and multi-hop networks that operate without the benefit of any existing infrastructure except for the nodes themselves. A MANET network cloud is composed of autonomous, potentially mobile and wireless nodes that may be connected at the edges to the fixed, wired internet. In this paper, we study different mobility models proposed in the recent research literature and their performance of routing protocols Bellman Ford, Dynamic Source Routing (DSR), Optimized Link State Routing (OLSR) and Distance Routing Effect Algorithm for Mobility (DREAM) by varying no. of nodes and mobility in terms of three metrics viz. Packet Delivery Ratio (PDR), end to end delay, and Throughput The simulation is carried out on NS -3 Simulator. The effort allows a fair comparison of the capabilities and limitations of different types of mobility patterns and their suitability for contemporary MANET routing protocols. In this study we have considered three mobility scenarios: Random Waypoint, Group Mobility and Freeway Models. These three Mobility Models are selected to represent possibility of practical application in future.
\end{abstract}

Keywords: MANET, Bellman Ford, DSR, OLSR, DREAM, Random waypoint Mobility, Group Mobility, Freeway Mobility Models

\section{INTRODUCTION}

Mobile Ad-Hoc Network (MANET) [1] is a collection of wireless nodes that dynamically create a wireless network among them without any infrastructure or centralized administration. Since no fixed infrastructure or centralized administration is available, these networks are self-organized and end-to-end communication may require routing information via several intermediate nodes. Nodes can connect each other randomly and forming arbitrary topologies. Each node in MANET acts both as a host and as a router to forward messages for other nodes that are not within the same radio range. The primary challenge in building a Mobile Ad hoc Network is equipping each device to continuously maintain the information required to route traffic.

To thoroughly and systematically study a new Mobile Ad hoc Network protocol, it is important to simulate this protocol and evaluate its protocol performance. Protocol simulation has several key parameters, including mobility model and communicating traffic pattern, among others. In this chapter and the next chapter we focus on the analysis and modeling of mobility models. We are also interested in studying the impact of mobility on the performance of MANET routing protocols. We present a survey of the status, limitations and research challenges of mobility modeling in this chapter.

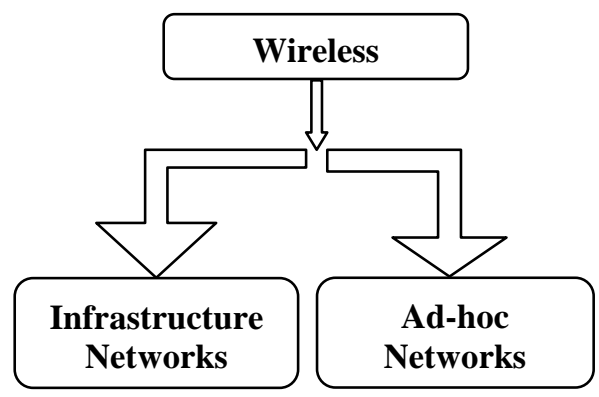

Figure 1: Wireless Networks Categories 


\section{International Advanced Research Journal in Science, Engineering and Technology}

Vol. 6, Issue 3, March 2019

Two variations of mobile wireless network are: infrastructure network and infrastructure less network. Infrastructure network has bridges, known as base stations [3]. These networks communicate with the nearest base station which lies within the range. The other variation is infrastructures less network, which are also called as Mobile ad hoc network (MANET). An ad hoc network [1] is a collection of mobile nodes forming a temporary network without the aid of any centralized administration or standard support services regularly available on conventional networks. Mobile Ad hoc networks (MANETs) are of much interest to both the research community and the military because of the potential to establish a communication network in any situation that involves emergencies. Examples are military deployment in hostile environment, search-and-rescue operations and several types of police and military operations. The advantages of such network are flexibility, rapid deployment, robustness, and inherent support for mobility.

The mobility model is designed to describe the movement pattern of mobile users, and how their location, velocity and acceleration change over time. Since mobility patterns may play a significant role in determining the protocol performance, it is desirable for mobility models to emulate the movement pattern of targeted real life applications in a reasonable way. Otherwise, the observations made and the conclusions drawn from the simulation studies may be misleading. Thus, when evaluating MANET protocols, it is necessary to choose the proper underlying mobility model. For example, the nodes in Random Waypoint model behave quite differently as compared to nodes moving in groups [1]. It is not appropriate to evaluate the applications where nodes tend to move together using Random Waypoint model. Therefore, there is a real need for developing a deeper understanding of mobility models and their impact on protocol performance.

In the previous studies on mobility patterns in wireless cellular networks[3][4], researchers mainly focus on the movement of users relative to a particular area (i.e., a cell) at a macroscopic level, such as cell change rate, handover traffic and blocking probability. However, to model and analyze the mobility models in MANET, we are more interested in the movement of individual nodes at the microscopic-level, including node location and velocity relative to other nodes Over the last few years, a number of routing protocols have been anticipated and enhanced to well route data packets among two nodes in MANETs [3]. It is not clear how those different protocols perform under different environments like that a protocol may be the best in one network configuration but the worst in another. The main aim of this paper is:

- Describing the detailed understanding of ad hoc routing protocols.

- Implementing the Mobility models

- Analyze and compare the performance of routing protocols under different mobility models. The purpose of this research is to provide understanding of how mobility affects routing in ad hoc networks and how to quantize those effects.

The rest of the paper is organized as follows. In following section, we first present an entity model named Random Way Point mobility model [4] and Freeway mobility model, called Reference Point Group Mobility model(RPGM)[5]. And further briefly discusses the MANET routing protocols description and the functionality of the two familiar reactive routing protocols AODV and DSR. Further it shows the simulation results and performance comparison of the two above said routing protocols. Finally, it concludes with the comparisons of the overall performance of two reactive routing protocols AODV and DSR based on metrics like packet delivery ratio (PDR), the average end-to-end delay and Throughput.

In Fig. 2 we provide a categorization for various mobility models into several classes based on their specific mobility characteristics. For some mobility models, the movement of a mobile node is likely to be affected by its movement history. We refer to this type of mobility model as mobility model with temporal dependency. In some mobility scenarios, the mobile nodes tend to travel in a correlated manner. We refer to such models as mobility models with spatial dependency. Another class is the mobility model with geographic restriction, where the movement of nodes is bounded by streets, freeways or obstacles. 


\section{International Advanced Research Journal in Science, Engineering and Technology}

Vol. 6, Issue 3, March 2019

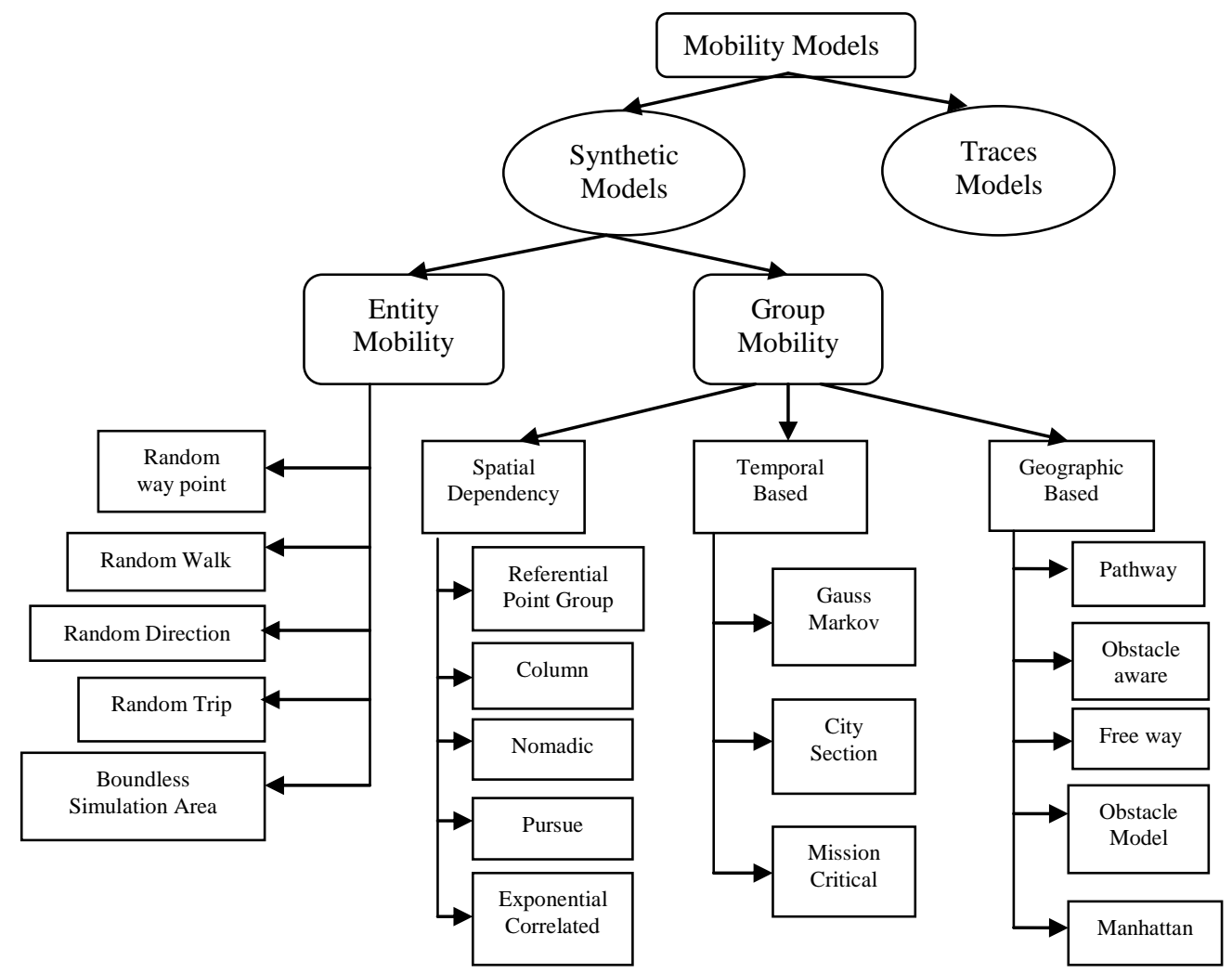

Figure 2: The categories of mobility models in Mobile Ad hoc Network

\section{MOBILITY MODELS}

Different mobility models can be differentiated according to their spatial and temporal dependencies.

Spatial dependency: It is a measure of how two nodes are dependent in their motion. If two nodes are moving in same direction then they have high spatial dependency.

Temporal dependency: It is a measure of how current velocity (magnitude and direction) are related to previous velocity. Nodes having same velocity have high temporal dependency.

\section{A. Random Waypoint Mobility Model}

The random way point model [4] is the simplest model but still the most widely used model to evaluate the performance of MANETs. The random way point model includes pause time between changes in direction and/or speed[7]. As a Mobile Node begins to move, it stays in one location for a certain period of time i.e. pause time. Once the pause time is elapsed, the Mobile node randomly chooses the next destination in the simulation area and selects a random speed uniformly distributed between [minspeed, maxspeed] and travels with a speed $\mathrm{v}$ which is uniformly chosen between the interval $(0, \mathrm{Vmax})$. Then, the Mobile Node continues its journey toward the newly selected destination at the chosen speed. As the mobile node arrives at the destination, it stays again for the specified pause time before continuing the process. The Random Waypoint Mobility Model is very widely used in simulation studies of MANET. As described in the performance measures in mobile ad hoc networks are affected by the mobility model used. Figures 3-5 illustrate examples of a topography showing the movement of nodes for Random Mobility Model.

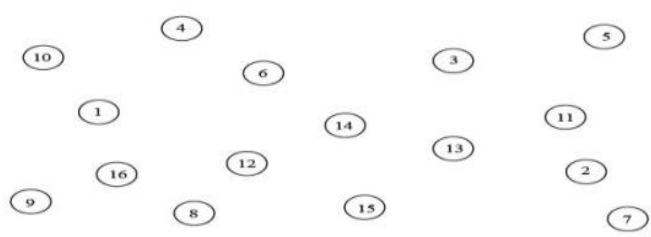

Figure 3. Topography showing the movement of nodes for Random mobility model.

\section{B. Random Point Group Mobility Model}

Random Point Group Mobility (RPGM) [5] Model described as another way to simulate group behaviour, The RPGM Group mobility may be used in rescue operations and military battlefield applications, where the commander 
and soldiers form a logical group. In reference point group mobility model, simulate group behaviour, where each node belongs to a group where every node follows a logical centre (group leader) that determines the group's motion behaviour. The nodes in a group are randomly distributed around a reference point. Each node uses their own mobility model and is then combined to the reference point, which directs them in the direction of group. The movement of a group leader determines the movement of the members of that group. This generic description of group mobility can be used to create diversity of models for different types of mobility applications. Given below is example topography showing the movement of nodes for Random Point Group Mobility Model. The scenario contains sixteen nodes with Node 1 and Node 9 as group leaders.
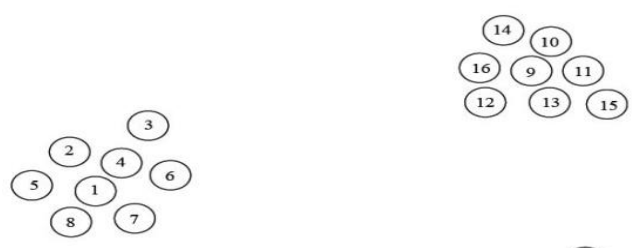

Figure 4. Topography showing the movement of nodes Random point group mobility

\section{Freeway Mobility Model}

This model emulates the motion behaviour of mobile nodes on a freeway. It can be used in exchanging traffic status or tracking a vehicle on a freeway. Each mobile node is restricted to its lane on the freeway. The velocity of mobile node is temporally dependent on its previous velocity. Given below is the example topography showing the movement of nodes for Freeway Mobility Model with twelve nodes.

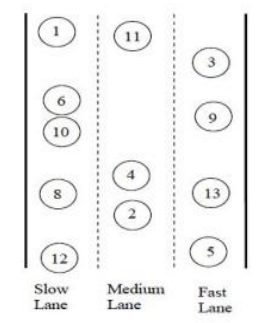

Figure 5. Topography showing the movement of nodes for Freeway mobility model.

\section{DESCRIPTION OF ROUTING PROTOCOLS}

\section{A. Bellman Ford Routing}

Bellman-Ford Routing Algorithm, also known as Ford-Fulkerson Algorithm, is used as an algorithm by distance vector routing protocols such as RIP, BGP, ISO IDRP, and NOVELL IPX. Routers that use this algorithm will maintain the distance tables, which tell the distances and shortest path to sending packets to each node in the network. The information in the distance table is always updated by exchanging information with the neighbouring nodes. The number of data in the table equals to that of all nodes in networks (excluded itself). The columns of table represent the directly attached neighbours whereas the rows represent all destinations in the network. Each data contains the path for sending packets to each destination in the network and distance/or time to transmit on that path. The Measurements in this algorithm are the number of hops, latency, the number of outgoing packets, etc.

\section{B. Optimized Link State Routing}

Optimized Link State Routing Protocol is based on link state algorithm. Being a proactive routing protocol, it has an advantage of having the route immediately available within the standard routing table when needed. Due to optimization nature minimum flooding duplication occurs in highly connected network. Each node in the network selects a set of neighbouring nodes to retransmit the packets and this set of nodes is called multipoint relays of that node. Instead of pure flooding the OLSR protocol employs Multipoint Relay (MPR) in network to reduce the possible overhead, flooding of broadcast and time interval for control message transmission. Only MPRs forward the control packets in such a way that information should reach entire network and these MPRs are responsible for declaring LS information. Each node periodically broadcasts a list of its one hop neighbours to select the MPRs with the help of hello message. Route calculations are done by MPR from source to destination node. OLSR supports three mechanisms: neighbour sensing, efficient flooding of control traffic and sufficient topology information. OLSR uses two types of control message: Hello and Topology Control (TC). Hello messages are used to find the information about 


\section{International Advanced Research Journal in Science, Engineering and Technology}

Vol. 6, Issue 3, March 2019

the link status and node's neighbours while TC messages are used for broadcasting information about own advertised neighbours includes at least the MPR selector list.

This MPR selector is obtained from HELLO packets sending between in neighbour nodes. These routes are built before any source node intends to send a message to a specified destination In order to exchange the topological information; the Topology Control (TC) message is broadcasted throughout the network. Each node maintains the routing table in which routes for all available destination nodes are kept. Control traffic in OLSR is exchanged through two different types of messages: "HELLO" and "TC" messages. HELLO messages are exchanged periodically among neighbour nodes, in order to detect links to neighbours, to detect the identity of neighbours and to signal MPR selection. TC messages are periodically flooded to the entire network, in order to signal link-state information to all nodes. The best working environment for OLSR protocol is a dense network, where the most communication is concentrated between a large numbers of nodes.

In figures 6(a) and 6(b) there is an illustrative example what is the cost difference between broadcast by flooding and by multipoint relays.
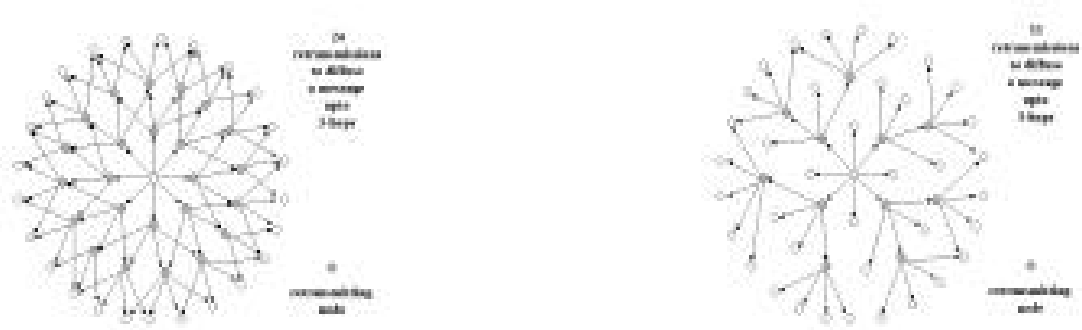

Figure 6: Diffusion of broadcast message using pure flooding (a) and multipoint relays (b)

\section{Dynamic Source Routing}

DSR is a reactive protocol based on source routing concept that requires each packet to carry the full address (every hop in the route) from source to destination. It is based on On-demand mechanism of route discovery and route maintenance. An advantage of DSR protocol is that nodes can store multiple routes in their route cache. Source node can check its route cache for a valid route before initiating route discovery, and if a valid route is found there is no need for route discovery. On the other hand, if a node does not have such a route, it initiates route discovery by broadcasting a RREQ packet. The RREQ packet contains the address of the destination along with address of source, a route record field and a unique identification number. Once the RREQ reaches either the destination or a node that knows a route to destination, it responds with a RREP along with the reverse of the route collected by the RREQ. A failed link is detected by either actively monitoring acknowledgements or passively running in promiscuous mode, overhearing that packet is forwarded by neighbouring node. The failed link is notified to the source node with RERR packet. The source node can use other known route to destination node or the process of route discovery is initiated again to find new route to destination. Another thing is to be noted that it does not require hello message exchanges, therefore nodes can enter sleep node to conserve their power. Also saves a considerable amount of bandwidth in the network.

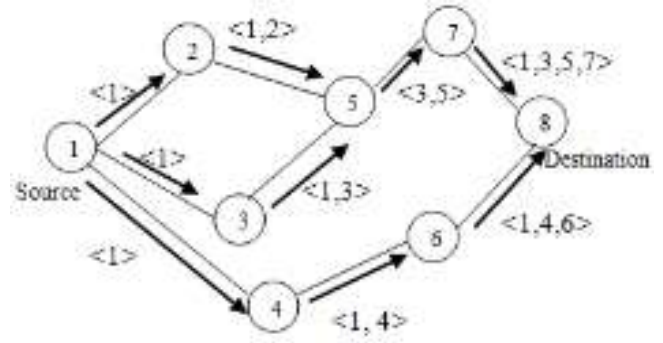

Figure 7(A): Route discovery

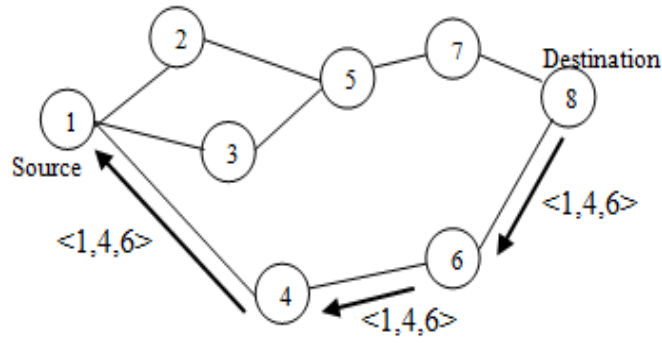

Figure 7(B): Route reply

For route discovery a source node broadcasts a route request packet to its neighbouring nodes as shown in Fig 7(A). In Fig. 7(B) a route reply message is sent back if the request packet reaches either destination nodes or intermediate nodes having active route to the destination nodes. 


\section{International Advanced Research Journal in Science, Engineering and Technology}

Vol. 6, Issue 3, March 2019

\section{Distance Routing Effect Algorithm for Mobility}

DREAM is a multi-path, location-aware routing protocol. In DREAM, each node knows its geographical coordinates through a Global Positioning System (GPS). The coordinates are periodically exchanged between each node and stored in a routing table. The advantage of exchanging location information compared to link state or distance vector information where complete information are exchanged is less bandwidth consumption resulting in good scalability of this protocol.

\section{SIMULATION ENVIORMENT}

NS3 is open source discrete-event network simulator and improves simulation credibility. The NS-3 project, started in 2006, is an open-source project developing NS-3. NS3 is not backward compatible with NS2, built from the scratch to replace NS2. NS2 and NS3 are both written with the help of C++ but NS3 does not support NS2 APIs. Ns-3 is free software, licensed under the GNU GPLv2 license. It will rely on the ongoing contributions of the community to develop new models, debug or maintain existing ones, and share results some models have been ported from NS2 to NS3. NS3 is written in C++, with optional python bindings. NetAnim is GUI based network simulator used for NS3. It is stand-alone program that uses XML trace files to display the simulation graphically. It is based on Qt4 GUI toolkit.

A. $\mathrm{C}++$ : $\mathrm{C}++$ used for implementation of simulation and core model. Ns-3 is built as a library which may be statically or dynamically linked to a C++ main program. These libraries define the start of simulation and simulation topology.

B. Python: C++ wrapped by Python. Python programs to import an "ns3" module. The component diagram of ns3 is given in following figure.

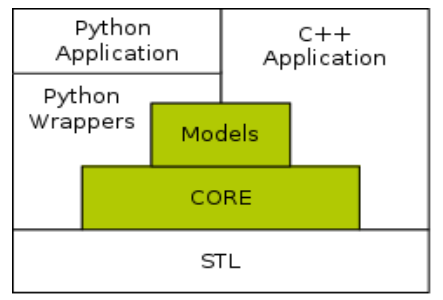

Figure 8: Architecture of NS3

\section{RESULTS}

Three performance metrics are used for measuring the performance of Bellman Ford, DSR, OLSR and DREAM Routing Protocols. The simulation results are shown in the form of graph that represents (i) Packet Delivery Ratio, (ii) Average End to End Delay and (iii) Throughput.

\section{A. Packet Delivery Ratio:}

Number of Data Packets Delivered over Number of Data Packets Generated. "Number of Data Packets Delivered" is the total number of received data packets by destinations; "Number of Data Packets Generated" is the total number of generated data packets by sources. Figure 9 (a), (b) and (c) shows the graph of Bellman Ford, DSR, OLSR and DREAM routing protocol for packet delivery ratio [9] between three mobility scenarios: Random Waypoint, Group Mobility and Freeway Models respectively.

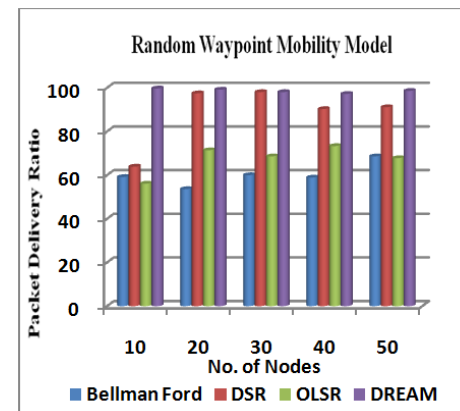

Figure 9 (a). PDR with

Random Way Point model

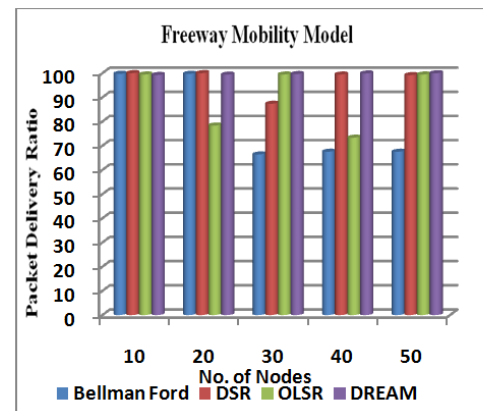

Figure 9 (b). PDR with

Freeway mobility model

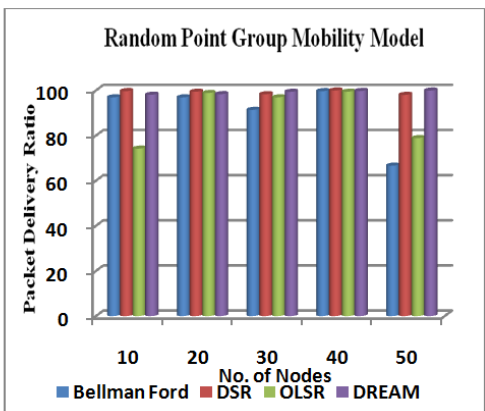

Figure 9 (c). PDR with

Random point group mobility model 


\section{International Advanced Research Journal in Science, Engineering and Technology}

Vol. 6, Issue 3, March 2019

\section{B. Average End to End Delay:}

Average packet delivery time from a source to a destination. First for each source-destination pair, an average delay for packet delivery is computed. Then the whole average delay is computed from each pair average delay. Figure 10 (a), (b) and (c) shows the graph of Bellman Ford, DSR, OLSR and DREAM routing protocol for average end to end delay between three mobility scenarios: Random Waypoint, Group Mobility and Freeway Models respectively.. Endto-end delay includes the delay in the send buffer, the delay in the interface queue, the bandwidth contention delay at the MAC, and the propagation delay.

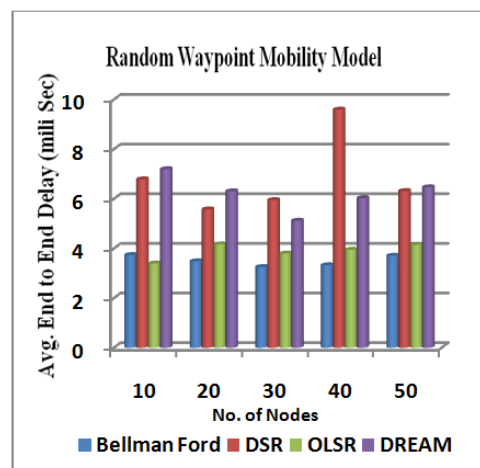

Figure 10 (a). End to End Delay with Random Way Point model

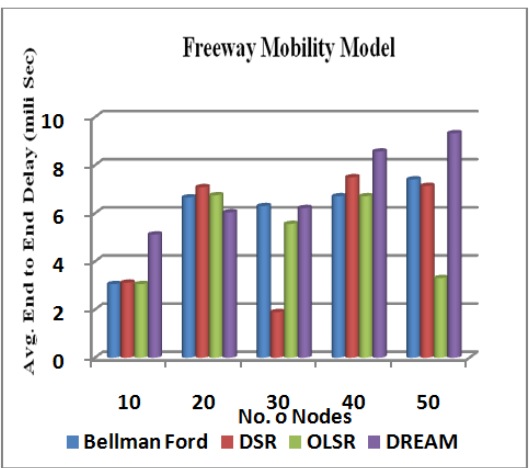

Figure 10 (b). End to End Delay with Freeway mobility model

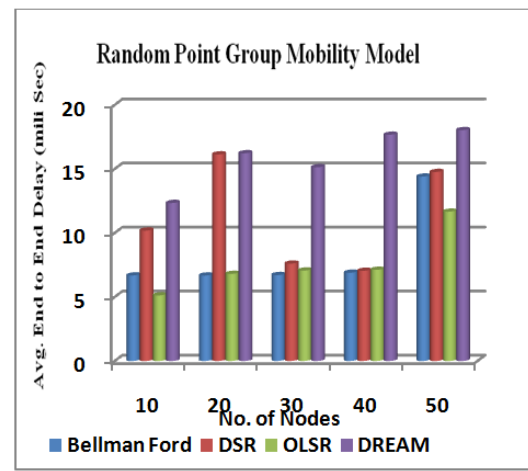

Figure 10 (c). End to End Delay with Random point group mobility model

\section{Throughput:}

Throughput is the number of packet that is passing through the channel in a particular unit of time. This performance metric show the total number of packets that have been successfully delivered from source node to destination node and it can be improved with increasing node density. Figure 11 (a), (b) and (c) shows the graph of Bellman Ford, DSR, OLSR and DREAM routing protocol for throughput between three mobility scenarios: Random Waypoint, Group Mobility and Freeway Models respectively.

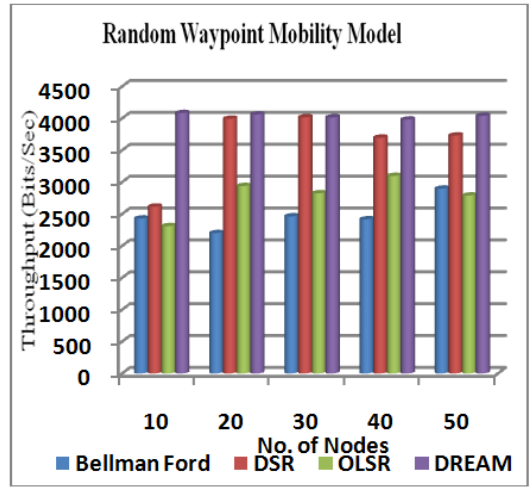

Figure 11 (a). Throuhput with Random Way Point model

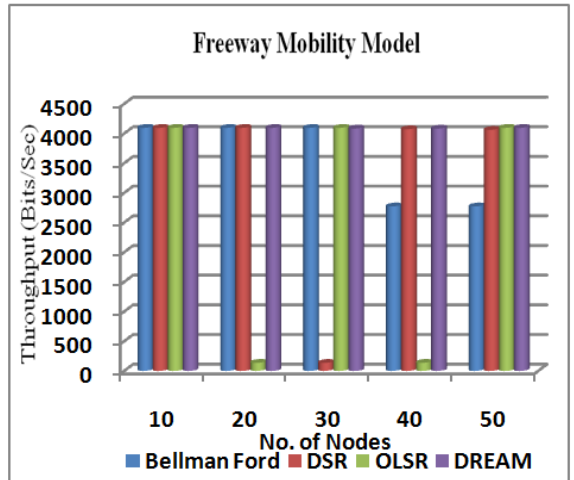

Figure 11 (b). Throuhput with Freeway mobility model

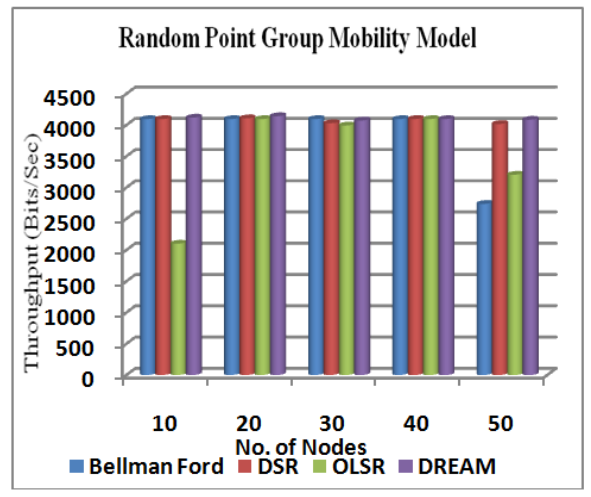

Figure 11 (c). Throuhput with Random point group mobility model

VI.

\section{CONCLUSION}

In this paper we have simulated the Bellman Ford, DSR, OLSR and DREAM routing protocols on NS version3 Simulator. The performance of the protocols was measured with respect to metrics like Packet delivery ratio, end to end delay and Throughput. Simulations were carried out with identical networks and running different protocols on the mobile node. The simulation is divided in three parts basis on the mobility model (random waypoint mobility, random point group mobility model and freeway mobility model). Here we conclude as:

1. DREAM performs well than DSR, Bellman Ford and OLSR (in reference to packet delivery ratio) if the node mobility is random waypoint and random point group mobility model.

2. Bellman Ford has performed well when the node mobility model is freeway mobility model.

3 . Packet delivery ratio is increases as the number of nodes increases.

4. Freeway mobility model is better than the other two mobility models in terms of Packet Delivery Ratio.

5. Bellman Ford performs better than DSR, DREAM and OLSR in terms of average end to end delay and random waypoint mobility model is better than random point group mobility model and freeway mobility model. 


\section{International Advanced Research Journal in Science, Engineering and Technology}

Vol. 6, Issue 3, March 2019

5. DSR and DREAM both have better Throughput than Bellman Ford and OLSR.

6. Random point group mobility is better in compare to freeway mobility model and random way point mobility model in terms of throughput.

For the above discussion we can say that all the routing protocols and mobility models have their own significance they all have their own advantages and disadvantages its depends upon the situation where we have to use. In some situation DREAM Routing is better and in some situation DSR is better. In some cases Random Waypoint mobility model is better and in some cases Random Point Group mobility model is better.

\section{VII. $\quad$ FUTURE SCOPE}

Future work may include same experiments for SRMP, SHARP, CGSR and ZRP, measuring the average end to end delay, packet delivery rate and drop ratio and the same experiments for different node mobility speed of the simulation and other mobility models. Another Future work is to perform the experiments for various different node migration speeds. We used the node mobility of $45 \mathrm{~km} / \mathrm{h}$ in our experiments this time. However, the node migration speed of $45 \mathrm{~km} / \mathrm{h}$ is just one of the possible velocities. Keeping the migration speed lower may lessen or rule out the cases of packets getting dropped even before routing information is updated. This may affect the simulation results and perhaps will bring out the strengths and weaknesses of different protocols unambiguously. Proactive protocols may give better performance for near stable environment. Performance of other routing protocol can be evaluated over various mobility models taking in to consideration number of average connected paths to gain greater insights into the relationship between them. Designing scenarios which depict real world applications more accurately can be designed through in-depth study of the application.

\section{ACKNOWLEDGEMENTS}

The author wishes to thank the reviewers and editors for their valuable suggestions and expert comments that help improve the quality as well as quantity of the paper. We would like to thank Dr. R.K. Mehta, Head of Department (IDE), MCAET, Ambedkar Nagar for providing continuous support.

\section{REFERENCES}

[1]. Nancy Garg, "Network Simulators: A Case Study", IJARCSSE, 5(1), January - 2015, pp. 651-655.

[2]. Yassine MALEH, DR. Abdellah Ezzati "Comparative Analysis of Routing Protocols AODV DSDV and DSR in MANET", IJCSMC, Vol. 3,Issue. 3, March 2014, ISSN 2320-088X.

[3]. Danish Vasan, "A Consolidated Analysis of MANET Routing Protocols", IJWCNT, 3(2), February - March $2014,30-34$.

[4]. Sanjeev Kumar, "Investigate and Improving Routing Mobility Based On Random Way Point Mobility Model Using FTP Traffic in MANET", IJARCSSE, 3(4), April - 2013, pp. 332-337.

[5]. Dr.S.S.Dhenakaran ${ }^{1}$, A. Parvathavarthini', “An Overview of Routing Protocols in Mobile Ad-Hoc Network", IJARCSSE, 3(2), February 2013, pp. 251-259.

[6]. Preeti Gaharwar ${ }^{1}$, Mr. Sunil R. Gupta ${ }^{2}$ Performance Comparison of AODV, DSDV \& DSR Routing Protocols in MANET IJARSSE, Volume 3, Issue 4, April 2013, ISSN: 2277 128X.

[7]. Preeti Gaharwar ${ }^{1}$, Mr. Sunil R. Gupta ${ }^{2}$ "Performance Comparison of Routing Protocols" IJACCE Vol. 2, Issue 4, April 2013, ISSN (Print): 2319-5940.

[8]. Somya Jain ${ }^{1}$, Deepak Aggarwal ${ }^{2}$ "Performance Evaluation of Routing Protocols for MAC Layer Models", IOSR-JCE, Volume 10, and Issue-4 (Mar.-Apr. 2013), PP 71-77, ISSN: 2278-8727.

[9]. Robinpreet Kaur \& Mritunjay Kumar Rai, A Novel Review on Routing Protocols in MANETs, Undergraduate Academic Research Journal (UARJ), ISSN : 2278 - 1129, Volume-1, Issue-1, 2012.

[10]. Dr. Kamaljit I. Lakhtaria, Analyzing Reactive Routing Protocols in Mobile Ad Hoc Networks, Int. J. Advanced Networking and Applications Volume:03 Issue:06 Pages:1416-1421 (2012) ISSN : 0975-0290.

[11]. C. Bettstetter, H. Hartenstein, and X. Perez-Costa, Stochastic Properties of the Random Waypoint Mobility Model, in ACM/Kluwer Wireless Networks, Special Issue on Modeling And Analysis of Mobile Networks, vol. 10, no. 5, Sept 2004.

[12]. F. Bai, A. Helmy, "A Survey of Mobility Modeling and Analysis in Wireless Adhoc Networks" in Wireless Ad Hoc and Sensor Networks, Kluwer Academic Publishers, 2004.

[13]. F. Bai, G. Bhaskara and A. Helmy," Building the Blocks of Protocol Design and Analysis Challenges and Lessons Learned from Case Studies on Mobile Adhoc Routing and Micro-Mobility Protocols", ACM Computer Communication Review, Vol.34, No.3, pp.57-70, 2004.

[14]. F. Bai, N. Sadagopan and A. Helmy, "IMPORTANT:A framework to systematically analyse the Impact of Mobility on Performance of Routing protocols for Adhoc Networks, IEEE INFOCOM, pp. 825-835, 2003.

[15]. David B. Johnson, David A. Maltz, Yih-Chun Hu, The Dynamic Source Routing (DSR) Protocol for Mobile Ad Hoc Networks.draft-ietfmanet-dsr-10.txt, July 2004.

[16]. David B. Johnson and David A. Maltz, "Dynamic Source Routing in Ad Hoc Wireless Networks", In Mobile Computing, edited by Tomasz Imielinski and Hank Korth, Chapter 5, pages 153-181, Kluwer Academic Publishers, 1996.

[17]. Biao Zhou, Kaixin Xu and Mario Gerla, "Group and Swarm Mobility Models for Ad Hoc Network Scenarios Using Virtual Tracks, In Proceedings of MILCOM'2004, Volume 1, pp. 289- 294, 1994.

[18]. User Manual for important Mobility Tool Generator in NS-3 Simulator. Release Date February 2010.

[19]. Mobility Generator (version1.0) from the site, http://nile.usc.edu/important/software.htm, February 2004. 


\title{
International Advanced Research Journal in Science, Engineering and Technology
}

\author{
Vol. 6, Issue 3, March 2019
}

\section{BIOGRAPHY}

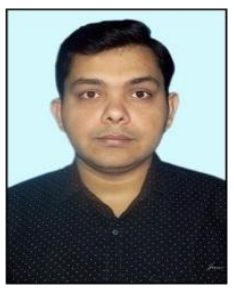

Ashish Gupta received the B.Tech. degree in Computer Science and Engineering from Inderprastha Engineering College Ghaziabad, affiliated with GAUTAM BUDDH TECHNICAL UNIVERSITY, Lucknow, India. After qualifying GATE, He completed M.Tech. in Computer Science and Engineering from Kanpur Institute of Technology, Kanpur, affiliated with UTTAR PRADESH TECHNICAL UNIVERSITY, LUCKNOW, India. Furthermore, he worked as an In-charge in department of Computer Science and Engineering at Mahamaya College of Agricultural Engineering and Technology, Ambedkar Nagar. His research interest is Mobile Computing and Software Engineering. 